\title{
The pathological profile of endometrial carcinoma in a cohort of Sri Lankan patients
}

\author{
N. K. Samarakoon, L. B. D. J. Siriwikum, S. K. Liyanage, B. A. G. G. Mahendra, R. M. U. S. \\ Rathnayake and S. J. de S Hewavisenthi \\ Department of Pathology, Faculty of Medicine, University of Kelaniya, Sri Lanka
}

DOI: http://doi.org/10.4038/jdp.v11i2.7720

\section{Introduction}

Endometrial cancer is the eighth most common cancer in Sri Lankan women. An understanding of the disease pattern of a local population would be useful in further management. The aim was to describe the pathological features and stage at presentation of endometrial carcinoma (EC) in a cohort of Sri Lankan patients.

\section{Methodology}

This is a retrospective study of 63 patients in the Western Province diagnosed with EC within the last five years. The macroscopic and microscopic features, grade and stage were obtained from the histopathology reports. The correlation between these parameters was assessed using the Pearson correlation coefficient ( $r$ ).

\section{Results}

The median age at presentation was 62 years (range 35-78). Out of the 63 patients, 38(60\%) were above 60 years, 52(83\%) were polypoidal tumours and $11(17 \%)$ showed diffuse thickening of the endometrium. Histological types were endometrioid in $56 / 63(89 \%)$, serous in $5 / 63(8 \%)$ and mixed endometrioid mucinous adenocarcinomas in 2/63(3\%). The EC comprised $36 / 63(57 \%)$ grade $1,14 / 63(22 \%)$ grade 2 and 13/63(21\%) grade 3 tumours. Lympho-vascular invasion (LVI), focal necrosis and squamoid differentiation was identified in 9/63 (14\%), 24/63 (38\%) and 15/63 (22\%) respectively. The number of patients presenting in stages $\mathrm{IA}$ and $\mathrm{IB}$ were equal being $24 / 63(38 \%)$. Stage $2,3 A, 3 B$ and $3 C$ tumours included 7/63(11\%), 5/63(8\%), 2/63(3\%) and $1 / 63(2 \%)$ respectively. No stage 4 tumours were recorded. There was a strong correlation between the stage and LVI( $r=0.609), \quad a$ moderate correlation between the grade and the LVI ( $r=0.412)$ and the grade and stage $(r=0.410)$. There was no correlation between the age and stage $(r=0.016)$.

\section{Conclusion}

Majority of endometrial carcinomas are of low grade and stage at diagnosis conferring a good prognosis and treatment outcome in our patients. Lympho-vascular invasion is significantly associated with a higher stage. 